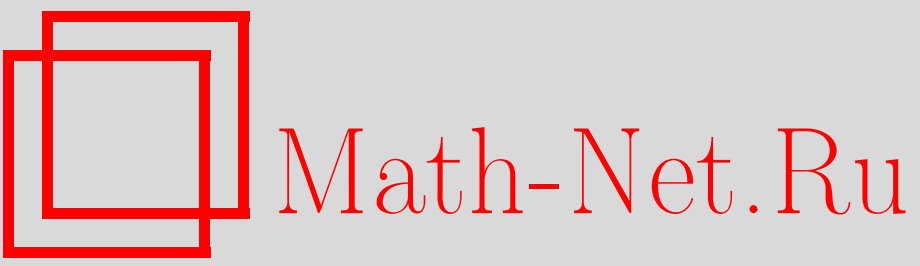

М. Е. Жуковский, Закон больших чисел для количества активированных частиц в модели эпидемии, Теория вероятн. и ее примен., 2013, том 58, выпуск 2, 235254

DOI: https://doi.org/10.4213/tvp4505

Использование Общероссийского математического портала Math-Net.Ru подразумевает, что вы прочитали и согласны с пользовательским соглашением http://www . mathnet.ru/rus/agreement

Параметры загрузки:

IP: 3.85 .73 .92

26 апреля 2023 г., $17: 49: 09$ 
ТЕОРИЯ ВЕРОЯТНОСТЕЙ

Том 58

И ЕЕ ПРИМЕНЕНИЯ

Выпуск 2

2013

(C) $2013 \Gamma$.

ЖУКОВСКИЙ М. Е.*

\title{
ЗАКОН БОЛЬШИХ ЧИСЕЛ ДЛЯ КОЛИЧЕСТВА АКТИВИРОВАННЫХ ЧАСТИЦ В МОДЕЛИ ЭПИДЕМИИ
}

\begin{abstract}
В последнее десятилетие целый ряд работ был посвящен изучению вероятностных свойств моделей эпидемии на графах. В данной работе мы рассматриваем обобщение модели, предложенной Ф. Машадо, Х. Машурианом и Х. Матзингером. Модель МашадоМашуриана-Матзингера служит интерпретацией процесса распространения вирусов в компьютерной сети. Мы предположили, что в каждый момент времени не только один узел сети может быть подвергнут заражению. В этой связи мы предлагаем более сложную модель, допуская каждый раз скачки нескольких частиц, причем количество таких частиц случайно. В работе доказан оптимальный вариант закона больших чисел для количества зараженных в рассмотренной модели эпидемии.
\end{abstract}

Ключевые слова и фразы: модель эпидемии, случайные блуждания, закон больших чисел, ветвящиеся процессы.

1. Введение. В последнее десятилетие целый ряд работ был посвящен изучению вероятностных свойств моделей эпидемии на графах (см., например, [1]-[7]). Дополнительный стимул это направление получило со стороны интернета, поскольку модели эпидемии играют существенную роль в изучении эволюции веб-графа (см., например, [8]). Моделирование интернета и изучение свойств этих моделей помогает, например, поисковым компаниям в улучшении качества ранжирования и в борьбе со спамом (см. [9]). Некоторые результаты для моделей эпидемий на графах оказываются полезными при исследовании химических реакций горения (см. [6]). Нас будет интересовать обобщение модели, предложенной Ф. Машадо, Х. Машурианом и Х. Матзингером в [10]. Эта модель тесно связана с классическим марковским процессом эпидемий SIR (см. [11]). Машадо, Машуриан и Матзингер рассматривали множество частиц, которые в момент времени $t=1$ находятся в точках множества $\left\{x_{1}, \ldots, x_{n}\right\}$. В точке $x_{1}$ располагается активная частица, а во всех остальных - неактивные (по одной частице в каждой точке). В любой момент времени $t \in \mathbf{N}$ ровно одна активная частица, номер которой имеет равномерное распределение на множестве номеров активных

*Московский государственный университет им. М. В. Ломоносова, Москва, Россия; e-mail: zhukmax@gmail.com 
частиц, имеющихся в момент $t$, совершает скачок в некоторую точку $x_{\xi(t)}$. При этом случайная величина $\xi(t)$ также равномерно распределена на $\{1, \ldots, n\}$. Если частица совершает скачок в точку с неактивной частицой, то эта частица активируется, а частица, совершившая скачок, остается активной. Активная частица умирает в том и только том случае, когда совершает скачок в точку, в которой находится или побывала ранее активная частица. В частности, если она совершает скачок в точку, где сама находится, то тоже погибает. Пусть величина $D_{n}(t)$ равна количеству неактивных частиц в момент $t$, а $\sigma_{n}-$ момент, в который погибает последняя активная частица. Для случайной величины $X_{n}=n-D_{n}\left(\sigma_{n}\right)$ в [10] установлена центральная предельная теорема. Заметим, что для обозначения различных типов частиц существует и другая терминология, которая использовалась и в [10].

Модель Машадо-Машуриана-Матзингера служит интерпретацией процесса распространения вирусов в компьютерной сети. Однако представляется более естественным предположить, что в каждый момент времени не только один узел сети может быть подвергнут заражению. В этой связи мы предлагаем более сложную модель, допуская каждый раз скачки нескольких частиц, причем количество таких частиц случайно. Целью работы является исследование предельного поведения по вероятности величин $X_{n}$, которые для новой модели имеют тот же смысл, что в [10], но описываются иначе. Рассмотрим множества $\left\{x_{1}(n), \ldots, x_{n}(n)\right\}$, $n \in \mathbf{N}$. Пусть, как и в модели работы [10], время $t$ дискретно. Будем считать, что в момент $t=1$ в каждой точке $x_{i}(n)$ находится одна частица с номером $i$. В точке $x_{1}=x_{1}(n)$ располагается активная частица, а во всех остальных располагаются неактивные. В каждый момент времени активная частица совершает скачок с вероятностью $p>0$ независимо от всех остальных активных частиц, при этом, если частица совершила скачок, то вероятность попадания в каждую точку равна $1 / n$. Иными словами, любая активная частица совершает случайное блуждание по вершинам полного графа. Схема взаимодействия активных и неактивных частиц та же, что в [10]. Однако если несколько частиц в один момент времени совершают скачки в одну и ту же точку с неактивной частицой, то все они остаются живы, а неактивная частица становится активной. Таким образом, с ненулевой вероятностью найдутся моменты времени, в которые в некоторых точках будет находиться по две и более активных частиц. Неактивные и активные частицы мы будем называть живьми.

Введем некоторые обозначения и формализуем описанную модель. Будем считать, что рассматриваемые случайные величины заданы на некотором вероятностном пространстве $(\Omega, \mathscr{F}, \mathbf{P})$. Пусть величина $A_{n}(t)$ равна количеству активных частиц в момент времени $t$, а $D_{n}(t)$, как и ранее, равна количеству неактивных частиц. Положим $\mathscr{F}_{n}(t)=$ 
$\sigma\left(D_{n}(t), A_{n}(t)\right)$. Количество частиц, совершивших скачок в момент времени $t$, обозначим $Y_{n}(t)$. Пусть, кроме того, $\mathscr{A}_{n}(t)=\left\{\zeta_{1}^{A}(t), \ldots, \zeta_{A_{n}(t)}^{A}(t)\right\}$, $\mathscr{D}_{n}(t)=\left\{\zeta_{1}^{D}(t), \ldots, \zeta_{D_{n}(t)}^{D}(t)\right\}, \mathscr{Y}_{n}(t)=\left\{\zeta_{1}^{Y}(t), \ldots, \zeta_{Y_{n}(t)}^{Y}(t)\right\}-$ соответственно множество активных частиц, множество неактивных частиц в момент $t$ и множество частиц, совершивших скачок в момент $t$. Выполнены равенства $\mathscr{A}_{n}(1)=\{1\}, \mathscr{D}_{n}(1)=\{2,3, \ldots, n\}, \mathbf{P}\left(\mathscr{Y}_{n}(1)=\{1\}\right)=p$, $\mathbf{P}\left(\mathscr{Y}_{n}(1)=\varnothing\right)=1-p$. Кроме того, $\mathscr{Y}_{n}(t) \subset \mathscr{A}_{n}(t)$ и справедливо следующее соотношение:

$$
\begin{gathered}
\forall t \in \mathbf{N} \forall a \in \mathbf{N} \forall k \in\{0,1, \ldots, a\} \forall\left\{i_{1}, \ldots, i_{k}\right\} \subset\{1, \ldots, a\} \\
\mathbf{P}\left(\mathscr{Y}_{n}(t)=\left\{\zeta_{i_{1}}^{A}(t), \ldots, \zeta_{i_{k}}^{A}(t)\right\} \mid A_{n}(t)=a\right)=p^{k}(1-p)^{a-k} .
\end{gathered}
$$

Определим также случайное подмножество $\mathscr{Z}_{n}^{+}(t)$ частиц, совершивших скачки в точки с неактивными частицами, распределенное по следующему закону:

$$
\begin{gathered}
\forall t \in \mathbf{N} \forall d, y \in\{1,2, \ldots, n\} \forall k \in\{0,1, \ldots, y\} \forall\left\{i_{1}, \ldots, i_{k}\right\} \subset\{1, \ldots, y\} \\
\mathbf{P}\left(\mathscr{Z}_{n}^{+}(t)=\left\{\zeta_{i_{1}}^{Y}(t), \ldots, \zeta_{i_{k}}^{Y}(t)\right\} \mid Y_{n}(t)=y, D_{n}(t)=d\right) \\
=\left(d n^{-1}\right)^{k}\left(1-d n^{-1}\right)^{y-k} .
\end{gathered}
$$

Пусть $\mathscr{Z}_{n}^{-}(t)=\mathscr{Y}_{n}(t) \backslash \mathscr{Z}_{n}^{+}(t), Z_{n}(t)=\left|\mathscr{Z}_{n}^{+}(t)\right|$, где $|X|-$ мощность множества $X$.

Для каждых $z, d \in \mathbf{N}$ и всех $\delta_{1}, \ldots, \delta_{d} \in\left\{x_{1}, \ldots, x_{n}\right\}$ определим случайные величины $\eta_{1}, \ldots, \eta_{z}$ на множестве $\left\{Z_{n}(t)=z, \mathscr{D}_{n}(t)=\right.$ $\left.\left\{\delta_{1}, \ldots, \delta_{d}\right\}\right\}$, распределение которых задано следующим образом:

$$
\begin{gathered}
\forall j \in\{1, \ldots, z\} \forall\left\{i_{1}, \ldots, i_{j}\right\} \subset\{1, \ldots, z\} \forall k_{1}, \ldots, k_{j} \in\{1,2, \ldots, d\} \\
\mathbf{P}\left(\eta_{i_{1}}=k_{1}, \ldots, \eta_{i_{j}}=k_{j} \mid Z_{n}(t)=z, \mathscr{D}_{n}(t)=\left\{\delta_{1}, \ldots, \delta_{d}\right\}\right)=d^{-j}
\end{gathered}
$$

Иными словами, $\eta_{1}, \ldots, \eta_{z}$ - это номера частиц, находящихся в момент $t$ в точках, в которые прыгают в этот же момент частицы из $\mathscr{Z}_{n}^{+}(t)$. Тогда для любого $\omega \in \Omega$ справедливы равенства

$$
\begin{aligned}
\mathscr{A}_{n}[t+1](\omega)= & \mathscr{A}_{n}[t](\omega) \cup \operatorname{Dif}\left(\left\{\zeta_{\eta_{1}(\omega)}^{D}(\omega), \ldots, \zeta_{\eta_{Z_{n}[t](\omega)}(\omega)}^{D}(\omega)\right\}\right) \\
& \backslash \mathscr{Z}_{n}^{-}[t](\omega), \\
\mathscr{D}_{n}[t+1](\omega)= & \mathscr{D}_{n}[t](\omega) \backslash \operatorname{Dif}\left(\left\{\zeta_{\eta_{1}(\omega)}^{D}(\omega), \ldots, \zeta_{\eta_{Z_{n}[t](\omega)}}^{D}(\omega)\right\}\right) .
\end{aligned}
$$

где $\operatorname{Dif}(X)$ - множество всех различных элементов в $X$.

Как и в модели «с одним скачком», рассмотренной в работе [10], $\sigma_{n}$ - момент, в который процесс $A_{n}(t)$ останавливается, т.е. $\sigma_{n}=$ $\min \left\{t \in \mathbf{N}: A_{n}(t)=0\right\}$. Если $A_{n}(t)>0$ для всех $t \in \mathbf{N}$, то положим 
$\sigma_{n}=0$. Мы изучаем предельное распределение количества частиц, которые были активированы в ходе процесса, т.е. случайной величины $X_{n}=n-D_{n}\left(\sigma_{n}\right)$ при $n \rightarrow \infty$.

Данная статья состоит из введения и двух частей. В первой из них формулируются основные результаты, а во второй даются их доказательства.

2. Основные результаты. Сформулируем основной результат работы, относящийся к закону больших чисел.

Теорема 1. Для любого $\gamma>3 / 4$ справедливо соотношение

$$
\left(X_{n}-\mathbf{E} X_{n}\right) n^{-\gamma} \stackrel{\mathbf{P}}{\longrightarrow} 0, \quad n \rightarrow \infty .
$$

Кроме того, для любого $\gamma<3 / 4$

$$
\liminf _{n \rightarrow \infty} \mathbf{P}\left(\left|X_{n}-\mathbf{E} X_{n}\right|>n^{\gamma}\right)>0
$$

3 а м е ч а н и е. Данный результат оптимален в том смысле, что (4) не выполнено при $\gamma<3 / 4$. Отметим также, что сходимость по вероятности в (4) нельзя заменить на сходимость почти наверное, как показывает следующее утверждение.

Лемма 1. Имеет место равенство

$$
\mathbf{P}\left(\lim _{n \rightarrow \infty} n^{-1}\left(X_{n}-\mathbf{E} X_{n}\right)=0\right)=0 .
$$

Из тождества (5), очевидно, следует, что сходимость по вероятности в (4) нельзя заменить на сходимость почти наверное ни при каком $\gamma \leqslant 1$.

3. Доказательства. Мы начнем с доказательства леммы 1 по двум причинам. Во-первых, оно простое. Во-вторых, интерес к доказательству сходимости по вероятности возрастает, когда нет сходимости почти наверное.

Д ок аз а те льс т в о ле м мы 1 . Очевидно, что $\mathbf{P}\left(X_{n}=1\right)=$ $p / n$. Учитывая независимость случайных величин $X_{1}, X_{2}, \ldots$, по лемме Бореля-Кантелли имеем $\mathbf{P}\left(\forall k \exists n \geqslant k: X_{n}=1\right)=1$. Но соотношение $\lim _{n \rightarrow \infty} n^{-1}\left(X_{n}(\omega)-\mathbf{E} X_{n}\right)=0$ не выполнено ни для какого $\omega \in$ $\left\{\forall k \exists n \geqslant k: X_{n}=1\right\}$, так как $\mathbf{E} X_{n}$ имеет порядок $n$ (в п. 3.4 будет показано, что существуют такие положительные числа $c$ и $n_{0}$, что для всех $n>n_{0}$ справедливо неравенство $\left.\mathbf{E} X_{n}>c n\right)$. Лемма доказана.

Д о к аз а т е л ь с т в о т е о р е мы 1 . Возьмем произвольные $\alpha \in(0,1 / 2), \delta \in(0, \infty)$. Разобьем отрезок $\left[1, \sigma_{n}\right]$ на два промежутка. По истечении первого периода времени $t_{\alpha}(n)=\left[(1 / 2-\alpha) \log _{1+p} n\right]$, где $[\cdot]$ - целая часть числа, с вероятностью, стремящейся к 1 , будет активировано $n^{1 / 2-\alpha}+o\left(n^{1 / 4+\delta}\right)$ частиц, а все остальные частицы будут 
живы. В п. 3.1 мы рассмотрим первый промежуток времени. Основной результат этого пункта мы сформулируем в виде леммы. Зависимость между рассматриваемыми в этом пункте случайными величинами достаточно слаба. Благодаря этому удается доказать упомянутую лемму. Несмотря на то что при $t>t_{\alpha}(n)$ зависимость усиливается, нам удалось построить независимые случайные величины, которые позволили использовать схемы суммирования независимых случайных величин и доказать теорему 1 . В п. 3.2 мы рассмотрим второй промежуток времени и получим оценки для отклонения случайных величин $A_{n}(t), D_{n}(t)$ от их условного математического ожидания $\mathbf{E}\left(\cdot \mid \mathscr{F}_{n}(t-1)\right)$ для каждого момента времени $t$ из этого промежутка. Наконец, в п. 3.3 мы выведем на основе результатов пп. $3.1,3.2$ рекуррентные соотношения для случайных величин $A_{n}(t)$ и $n-D_{n}(t)$, с помощью которых в п. 3.4 докажем первую часть утверждения теоремы 1 (про сходимость к нулю). Завершим доказательство теоремы в п. 3.5.

3.1. Первые скачки. Весь этот пункт мы посвятим доказательству сформулированной ниже леммы. Положим $B_{n}(t)=A_{n}(t)+D_{n}(t)$. Пусть $0<\alpha<1 / 2, \delta>0$.

Лемма 2. Имеем

$$
\lim _{n \rightarrow \infty} \mathbf{P}\left(\left|A_{n}\left(t_{\alpha}(n)\right)-n^{1 / 2-\alpha}\right|<n^{1 / 4+\delta}, B_{n}\left(t_{\alpha}(n)\right)=n\right)=1 .
$$

Кроме того, для любого $\mu>0$ при достаточно больших $n$ выполнено неравенство

$$
\begin{aligned}
\mathbf{P}\left(\operatorname { m i n } \left\{\left|A_{n}\left(t_{\alpha}(n)\right)-n^{1 / 2-\alpha}\right|, \mid\right.\right. & \left.\left.n-D_{n}\left(t_{\alpha}(n)\right)-n^{1 / 2-\alpha} \mid\right\}>n^{1 / 4-\alpha / 2-\delta}\right) \\
> & \frac{1}{2}-\mu .
\end{aligned}
$$

Д о к а з а т е л ь с т в о. Пусть $c \in(0,(-\ln p) /(2(1-\ln p)))$. При $n \geqslant 2$ положим

$$
\begin{aligned}
y(n)=\ln ^{-1 / 2}\left(p c \ln ^{2} \ln n\right), & J_{n}(t)=\mathbf{N} \cap\left[\left[\ln ^{2} \ln n\right], t\right], \\
t_{\alpha}^{-}(n)=\left[t_{\alpha}(n)(1-y(n))-\ln ^{2} \ln n\right], & t_{\alpha}^{+}(n)=\left[t_{\alpha}(n)(1+y(n))+\ln ^{2} \ln n\right] .
\end{aligned}
$$

Для функции $f: \mathbf{N} \rightarrow \mathbf{N}$ введем обозначение $\Delta f(i)=f(i+1)-f(i)$. Определим вспомогательные события:

$$
\begin{aligned}
W_{n}^{1} & =\left\{\sharp\left\{t \in\left\{1, \ldots,\left[\ln ^{2} \ln n\right]\right\}: Y_{n}(t)>0\right\} \geqslant p c \ln ^{2} \ln n\right\}, \\
W_{n}^{2}(t) & =\left\{B_{n}(t)=n\right\}, \quad \widetilde{W}_{n}^{1}=W_{n}^{1} \cap W_{n}^{2}\left(\left[\ln ^{2} \ln n\right]\right), \\
W_{n}^{3}(t) & =\left\{\forall i \in J _ { n } ( t ) \left(( A _ { n } ( i ) > 1 ) \wedge \left(\left|\Delta A_{n}(i)\left(A_{n}(i) p\right)^{-1}-1\right|\right.\right.\right. \\
\leqslant & \left.\left.\left.\ln ^{-1 / 2}\left(A_{n}(i)\right)\right)\right)\right\},
\end{aligned}
$$




$$
\begin{array}{r}
\widetilde{W}_{n}^{3}(t)=\left\{\forall i \in J _ { n } ( t ) \left(( A _ { n } ( i ) > 1 ) \wedge \left(\left|Y_{n}(i)\left(A_{n}(i) p\right)^{-1}-1\right|\right.\right.\right. \\
\left.\left.\left.\leqslant \ln ^{-2}\left(A_{n}(i)\right)\right)\right)\right\}, \\
W_{n}^{4}(t)=\left\{\forall i \in J _ { n } ( t ) \left(( Y _ { n } ( i ) > 1 ) \wedge \left(\left|\Delta A_{n}(i)\left(Y_{n}(i)\right)^{-1}-1\right|\right.\right.\right. \\
\left.\left.\left.\leqslant \ln ^{-2 / 3}\left(A_{n}(i)\right)\right)\right)\right\} .
\end{array}
$$

Здесь $\sharp \mathscr{A}$ обозначает мощность конечного множества $\mathscr{A}$. В дальнейшем мы покажем, что мера $\mathbf{P}$ каждого из этих множеств при $t \leqslant t_{\alpha}^{+}(n)$ стремится к 1 при $n \rightarrow \infty$. Для любого $i \in \mathbf{N}$ рассмотрим множества

$$
\begin{aligned}
& W_{n}^{3, i}=\left\{\left(A_{n}(i)>1\right) \wedge\left(\left|Y_{n}(i)\left(A_{n}(i) p\right)^{-1}-1\right| \leqslant \ln ^{-2}\left(A_{n}(i)\right)\right)\right\}, \\
& W_{n}^{4, i}=\left\{\left(Y_{n}(i)>1\right) \wedge\left(\left|\Delta A_{n}(i)\left(Y_{n}(i)\right)^{-1}-1\right| \leqslant \ln ^{-2 / 3}\left(A_{n}(i)\right)\right)\right\}
\end{aligned}
$$

и установим некоторые их свойства.

Р1. При $t>\ln ^{2} \ln n$ имеем

$$
\mathbf{P}\left(W_{n}^{3}(t) \cap W_{n}^{2}(t) \mid \widetilde{W}_{n}^{1}\right)=\mathbf{P}\left(W_{n}^{3}(t) \cap W_{n}^{2}(t) \cap W_{n}^{1}\right)\left(\mathbf{P}\left(\widetilde{W}_{n}^{1}\right)\right)^{-1} .
$$

Р2. При достаточно больших $n$ и $t>\ln ^{2} \ln n$ выполнено неравенство

$$
\mathbf{P}\left(W_{n}^{3}(t) \mid \widetilde{W}_{n}^{1}\right) \geqslant \mathbf{P}\left(\widetilde{W}_{n}^{3}(t) \cap W_{n}^{4}(t) \mid \widetilde{W}_{n}^{1}\right) .
$$

Р3. Для любого $t>\ln ^{2} \ln n$ справедливы равенства

$$
\bigcap_{i=\left[\ln ^{2} \ln n\right]}^{t} W_{n}^{3, i}=\widetilde{W}_{n}^{3}(t), \bigcap_{i=\left[\ln ^{2} \ln n\right]}^{t} W_{n}^{4, i}=W_{n}^{4}(t) .
$$

Свойства Р2 и Р3 очевидны. Свойство Р1 выполнено благодаря убыванию семейства $\left\{W_{n}^{2}(t), t \in \mathbf{N}\right\}$. Семейство $\{W(t) \in \mathscr{F}, t \in T \subset \mathbf{R}\}$ называется убывающим, если из того, что $t_{1}, t_{2} \in T, t_{1}<t_{2}$, следует, что $W\left(t_{1}\right) \supseteq W\left(t_{2}\right)$.

Доказательство того, что рассмотренные множества имеют достаточно большую меру, проведем следующим образом. Вначале мы покажем, что вероятность $\mathbf{P}\left(\widetilde{W}_{n}^{1}\right)$ стремится к 1, затем мы установим аналогичное свойство для вероятностей $\mathbf{P}\left(W_{n}^{2}\left(t_{\alpha}^{+}(n)\right) \mid \widetilde{W}_{n}^{1}\right)$ и $\mathbf{P}\left(\widetilde{W}_{n}^{3}\left(t_{\alpha}^{+}(n)\right) \mid \widetilde{W}_{n}^{1}\right)$, после чего сделаем вывод, что вероятность $\mathbf{P}\left(W_{n}^{4}\left(t_{\alpha}^{+}(n)\right) \cap \widetilde{W}_{n}^{3}\left(t_{\alpha}^{+}(n)\right) \cap \widetilde{W}_{n}^{1}\right)$ также стремится к 1 . Если описанные сходимости имеют место, то из свойств $\mathrm{P} 1$ и $\mathrm{P} 2$ следует равенство

$$
\lim _{n \rightarrow \infty} \mathbf{P}\left(W_{n}^{1} \cap W_{n}^{2}\left(t_{\alpha}^{+}(n)\right) \cap W_{n}^{3}\left(t_{\alpha}^{+}(n)\right)\right)=1 .
$$

Итак, начнем с $\mathbf{P}\left(\widetilde{W}_{n}^{1}\right)$. Для $0 \leqslant p \leqslant 1, n \in \mathbf{N}, k \in \mathbf{N}, k>$ $n p$ справедливо неравенство Чернова (см. [12]) $\sum_{i \geqslant k} C_{n}^{i} p^{i}(1-p)^{n-i} \leqslant$ 
$\exp \left(-(n p-k)^{2} /(2 n p)\right)$. Поэтому для некоторого $\epsilon>0$ и любого $\delta>0$ справедливы соотношения

$$
\begin{gathered}
\mathbf{P}\left(\overline{W_{n}^{1}}\right) \leqslant \sum_{i=0}^{[p c \ln 2 \ln n]} C_{\left[\ln ^{2} \ln n\right]}^{i}(1-p)^{\left[\ln ^{2} \ln n\right]-i}=O\left(e^{-\epsilon \ln ^{2} \ln n}\right), \\
\mathbf{P}\left(\overline{W_{n}^{2}\left(\left[\ln ^{2} \ln n\right]\right)}\right) \leqslant p \ln ^{2} \ln n\left(2^{\ln ^{2} \ln n} n^{-1}\right)=o\left(n^{-1+\delta}\right) .
\end{gathered}
$$

Следовательно, $\lim _{n \rightarrow \infty} \mathbf{P}\left(\widetilde{W}_{n}^{1}\right)=1$. Обратимся теперь к $\mathbf{P}\left(W_{n}^{2}\left(t_{\alpha}^{+}(n)\right) \mid \widetilde{W}_{n}^{1}\right)$. Покажем, что за время $t_{\alpha}^{+}(n)$ будет активировано не более $n^{1 / 2-\beta}$ частиц для любого $\beta \in(0, \alpha)$. Для всех положительных $\varepsilon<1-p$, $\delta<1-(1 / 2)(p+\varepsilon)$ имеем

$$
\begin{aligned}
& \mathbf{P}\left(n-D_{n}\left(t_{\alpha}^{+}(n)\right)>n^{1 / 2-\beta} \mid \widetilde{W}_{n}^{1}\right) \\
& \leqslant 1-\mathbf{P}\left(\forall t \in J _ { n } ( t _ { \alpha } ^ { + } ( n ) ) \left(\left(-\Delta D_{n}(t) \leqslant A_{n}(t) p(1+\varepsilon)\right)\right.\right. \\
& \left.\left.\wedge\left(\Delta A_{n}(t) \geqslant 0\right)\right) \mid \widetilde{W}_{n}^{1}\right) \\
& \leqslant \sum_{t=\left[\ln ^{2} \ln n\right]}^{t_{\alpha}^{+}(n)}\left(\mathbf{P}\left(\Delta A_{n}(t)<0 \mid \mathscr{W}_{n}(t) \cap \widetilde{W}_{n}^{1}\right)\right. \\
& \left.+\mathbf{P}\left(-\Delta D_{n}(t)>A_{n}(t) p(1+\varepsilon) \mid \mathscr{W}_{n}(t) \cap \widetilde{W}_{n}^{1}\right)\right) \\
& \leqslant t_{\alpha}^{+}(n) \max _{t \in J_{n}\left(t_{\alpha}^{+}(n)\right)}\left\{2 \mathbf{P}\left(\mathscr{W}_{n}^{0}(t) \mid \mathscr{W}_{n}^{1}(t)\right)\right. \\
& \left.\quad+\mathbf{P}\left(\overline{\mathscr{W}}_{n}^{0}(t) \cap\left\{\Delta A_{n}(t)>0\right\} \mid \mathscr{W}_{n}^{1}(t)\right)\right\} \\
& \leqslant t_{\alpha}^{+}(n) \max _{t \in J_{n}\left(t_{\alpha}^{+}(n)\right)}\left\{2 \mathbf{P}\left(\mathscr{W}_{n}^{0}(t) \mid \mathscr{W}_{n}^{1}(t)\right)\right. \\
& \left.\quad+\mathbf{P}\left(-\Delta D_{n}(t) \leqslant \frac{p+\varepsilon}{2} A_{n}(t) \mid \mathscr{W}_{n}^{1}(t)\right)\right\} \\
& \leqslant t_{\alpha}^{+}(n)\left(2 \exp \left(-\frac{1}{2} \varepsilon^{2} c \ln ^{2} \ln n\right)+\exp \left(-\left(1-\delta-\frac{p+\varepsilon}{2}\right)^{2} \frac{c \ln ^{2} \ln n}{2 p}\right)\right) \\
& =o\left((\ln n)^{-1}\right), \quad
\end{aligned}
$$

где

$$
\begin{gathered}
\mathscr{W}_{n}(t)=\bigcap_{i \in J_{n}(t) \backslash\{t\}}\left\{\left(-\Delta D_{n}(i) \leqslant A_{n}(i) p(1+\varepsilon)\right) \wedge\left(\Delta A_{n}(i) \geqslant 0\right)\right\}, \\
\mathscr{W}_{n}^{0}(t)=\left\{Y_{n}(t)>A_{n}(t)(p+\varepsilon)\right\}, \quad \mathscr{W}_{n}^{1}(t)=\mathscr{W}_{n}(t) \cap \widetilde{W}_{n}^{1} .
\end{gathered}
$$

Последнее неравенство в цепочке получено с помощью неравенства Чернова.

Тогда для любого $\beta \in(0, \alpha)$ приходим к оценке

$$
\mathbf{P}\left(W_{n}^{2}\left(t_{\alpha}^{+}(n)\right) \mid \widetilde{W}_{n}^{1}\right) \geqslant 1-\sum_{t=\left[\ln ^{2} \ln n\right]}^{t_{\alpha}^{+}(n)} \mathbf{P}\left(\Delta B_{n}(t)<0 \mid \widetilde{W}_{n}^{1}\right)=1+o\left(\ln ^{-1} n\right) .
$$


Перейдем к доказательству соотношения $\lim _{n \rightarrow \infty} \mathbf{P}\left(\widetilde{W}_{n}^{3}\left(t_{\alpha}^{+}(n)\right)\right)=1$. Пусть $t \leqslant t_{\alpha}^{+}(n), 0<\beta<\alpha, 1-n^{-1 / 2-\beta}<v=v(n)<1$. Тогда

$$
\begin{gathered}
\mathbf{P}\left(\zeta_{i}^{A}(t) \in \mathscr{Z}_{n}^{+}(t) \mid\left\{i \leqslant A_{n}(t)\right\} \cap\left\{D_{n}(i)=v n\right\} \cap W_{n}^{1} \cap W_{n}^{2}(t+1)\right) \\
=p v(1-p+p v)^{-1},
\end{gathered}
$$

при этом $p\left(1-n^{-1 / 2-\beta}\right) \leqslant p v(1-p+p v)^{-1}<p$. Поэтому в силу неравенства Чернова

$$
\begin{aligned}
& \mathbf{P}\left(\overline{W_{n}^{3, t}} \mid W_{n}^{1} \cap W_{n}^{2}(t+1)\right) \\
& \quad \leqslant \max _{m \in\left\{\left[p c \ln ^{2} \ln n\right], \ldots,\left[n^{1 / 2-\beta}\right]+1\right\}}\left\{2 e^{-p m / \ln ^{4} m}\right\}+o\left(\ln ^{-1} n\right)=o\left(\ln ^{-1} n\right) .
\end{aligned}
$$

Следовательно, согласно свойству Р3, для некоторой константы $C>0$ имеем

$$
\begin{aligned}
\mathbf{P} & \left(\widetilde{W}_{n}^{3}\left(x_{n}\right) \mid \widetilde{W}_{n}^{1}\right) \\
& \geqslant \mathbf{P}\left(\widetilde{W}_{n}^{3}\left(x_{n}\right) \mid W_{n}^{2}\left(t_{\alpha}^{+}(n)\right) \cap W_{n}^{1}\right) \mathbf{P}\left(W_{n}^{2}\left(t_{\alpha}^{+}(n)\right) \cap W_{n}^{1}\right)\left(\mathbf{P}\left(\widetilde{W}_{n}^{1}\right)\right)^{-1} \\
& \geqslant \mathbf{P}\left(W_{n}^{2}\left(t_{\alpha}^{+}(n)\right) \cap W_{n}^{1}\right)\left(\mathbf{P}\left(\widetilde{W}_{n}^{1}\right)\right)^{-1}-C \ln ^{-1 / 2} n \rightarrow 1, \quad n \rightarrow \infty .
\end{aligned}
$$

Осталось рассмотреть $\mathbf{P}\left(W_{n}^{4}\left(t_{\alpha}^{+}(n)\right) \cap \widetilde{W}_{n}^{3}\left(t_{\alpha}^{+}(n)\right) \cap \widetilde{W}_{n}^{1}\right)$. Семейства $\left\{W_{n}^{2}(t), t \in \mathbf{N}\right\},\left\{\widetilde{W}_{n}^{3}(t), t \in \mathbf{N}\right\}$ убывают, поэтому существуют такие числа $C_{1}, C_{2}, C_{3} \in(0, \infty)$, что

$$
\begin{gathered}
\mathbf{P}\left(\overline{W_{n}^{4}\left(t_{\alpha}^{+}(n)\right)} \cap W_{n}^{2}\left(t_{\alpha}^{+}(n)\right) \cap \widetilde{W}_{n}^{3}\left(t_{\alpha}^{+}(n)\right) \cap \widetilde{W}_{n}^{1}\right) \\
\leqslant \sum_{t=\left[\ln ^{2} \ln n\right]}^{t_{\alpha}^{+}(n)} \mathbf{P}\left(\overline{W_{n}^{4, t}} \cap W_{n}^{2}(t+1) \cap \widetilde{W}_{n}^{3}(t) \cap \widetilde{W}_{n}^{1}\right) \\
\leqslant t_{\alpha}^{+}(n) \max _{g \in G}\left\{e^{g\left(C_{1} g^{2} /\left(n-C_{1} g\right)\right)}\left(\frac{g}{n-C_{1} g}\right)^{C_{2} g / \ln g}\right\} \\
\leqslant t_{\alpha}^{+}(n) \max _{g \in G}\left\{e^{g\left(C_{1} g^{2} /\left(n-C_{1} g\right)-C_{3}\right)}\right\}=o\left(\frac{1}{\ln n}\right),
\end{gathered}
$$

где

$$
\begin{gathered}
G=\mathbf{N} \cap\left[p^{2} c \ln ^{2} \ln n\left(1-\ln ^{-2} \ln \ln n\right)\left(1-\ln ^{-2 / 3} \ln \ln n\right),\right. \\
\left.p n^{1 / 2-\alpha}\left(1+\ln ^{-1} n\right)\right] .
\end{gathered}
$$

Поэтому $\lim _{n \rightarrow \infty} \mathbf{P}\left(W_{n}^{4}\left(t_{\alpha}^{+}(n)\right) \cap \widetilde{W}_{n}^{3}\left(t_{\alpha}^{+}(n)\right) \cap \widetilde{W}_{n}^{1}\right)=1$. Равенство (7) доказано. 
Пусть $t_{n}$ - момент пересечения процессом $A_{n}(t)$ уровня $n^{1 / 2-\alpha}$. Тогда на множестве $\widetilde{W}_{n}^{1} \cap W_{n}^{2}\left(t_{\alpha}^{+}(n)\right) \cap W_{n}^{3}\left(t_{\alpha}^{+}(n)\right)$ значения такой случайной величины сосредоточены в интервале $\left(t_{\alpha}^{-}(n), t_{\alpha}^{+}(n)\right)$, так как

$$
\begin{aligned}
& 2^{\ln ^{2} \ln n}((1+p)(1+y(n)))^{t_{\alpha}^{-}(n)-\ln ^{2} \ln n} \\
& \quad<n^{1 / 2-\alpha}<p c \ln ^{2} \ln n((1+p)(1-y(n)))^{t_{\alpha}^{+}(n)-\ln ^{2} \ln n} .
\end{aligned}
$$

Иными словами, с вероятностью, стремящейся к 1 , при $t<t_{\alpha}^{-}(n)$ выполнено неравенство $n-D_{n}(t)<n^{1 / 2-\alpha}$, а при $t>t_{\alpha}^{+}(n)-$ неравенство $n-D_{n}(t)>n^{1 / 2-\alpha}$. Кроме того, на каждом шаге $t \in\left[t_{\alpha}^{-}(n), t_{\alpha}^{+}(n)\right]$ условная вероятность $\mathbf{P}\left(\cdot \mid \widetilde{W}_{n}^{1} \cap W_{n}^{2}(t+1) \cap W_{n}^{3}(t)\right)$ того, что количество новых активированных частиц отклоняется от математического ожидания более чем на $\sqrt[4]{n} \ln n$, равна $o(1 / n)$ в соответствии с неравенством Чернова. Поэтому для любого $\delta>0$ с вероятностью, стремящейся к 1 , справедливы неравенства

$$
\begin{aligned}
\mid n- & D_{n}\left(t_{\alpha}(n)\right)-n^{1 / 2-\alpha} \mid \\
& \leqslant n^{1 / 4} \ln n \exp \left(\ln (1+p)\left(2 \ln ^{2} \ln n+2 t_{\alpha}(n) y(n)\right)\right)<n^{1 / 4+\delta} .
\end{aligned}
$$

Положим $W_{n}^{-1}=\widetilde{W}_{n}^{1} \cap W_{n}^{2}\left(t_{\alpha}(n)-1\right) \cap W_{n}^{3}\left(t_{\alpha}(n)-1\right)$. Из центральной предельной теоремы Ляпунова (см., например, [13, с. 455]) вытекает, что

$$
\begin{aligned}
& \lim _{n \rightarrow \infty} \mathbf{P}\left(D_{n}\left(t_{\alpha}(n)\right)-\mathbf{E}\left(D_{n}\left(t_{\alpha}(n)\right) \mid \mathscr{F}_{n}\left(t_{\alpha}(n)-1\right)\right) \geqslant n^{1 / 4-\alpha-\delta} \mid W_{n}^{-1}\right)=\frac{1}{2}, \\
& \lim _{n \rightarrow \infty} \mathbf{P}\left(D_{n}\left(t_{\alpha}(n)\right)-\mathbf{E}\left(D_{n}\left(t_{\alpha}(n)\right) \mid \mathscr{F}_{n}\left(t_{\alpha}(n)-1\right)\right) \leqslant-n^{1 / 4-\alpha-\delta} \mid W_{n}^{-1}\right)=\frac{1}{2} .
\end{aligned}
$$

Таким образом, для любого $\mu>0$ при достаточно больших $n$ справедливо (6). Лемма доказана.

3.2. Второй период времени. Рассмотрим произвольный момент $t=t(n)$ из промежутка $\left(t_{\alpha}^{-}(n), \infty\right)$ и исследуем, как изменяется количество активных частиц в этот момент времени. Нас будет интересовать, какое количество частиц совершает скачки в точки с неактивными частицами, сколько всего частиц совершает скачки и как много окажется различных неактивных частиц, активированных в момент $t$. Текущий пункт мы посвятим доказательству того, что с вероятностью, стремящейся к 1 , выполнены приводимые в конце пункта неравенства (13) и (14).

Начнем с изучения распределения количества активированных частиц. Введем функции $s=s(n), u=u(n), \nu_{1}=\nu_{1}(n), \ldots, \nu_{s}=\nu_{s}(n)$, определенные для $n \in \mathbf{N}$ и принимающие натуральные значения. В дальнейшем $\nu_{1}, \ldots, \nu_{s}$ будут служить номерами частиц, совершивших 
скачки в точки с неактивными частицами в момент $t$. Пусть для некоторой положительной константы $C$ неравенство $u(n) \geqslant C n$ справедливо сразу для всех $n$. Пусть, кроме того, для каждого $n \in \mathbf{N}$ справедливы неравенства $\nu_{1}(n)<\cdots<\nu_{s}(n)$. Введем множество $\widetilde{\Omega}=\widetilde{\Omega}(n)=$ $\left\{\mathscr{Z}_{n}^{+}(t)=\left\{\zeta_{\nu_{1}}^{Y}, \ldots, \zeta_{\nu_{s}}^{Y}\right\}\right\} \cap\left\{D_{n}(t)=u\right\}$. Пусть $\xi\left(\nu_{i}\right)$ - случайные величины, равные на множестве $\widetilde{\Omega}$ индексам точек, в которые были совершены скачки частицами $\zeta_{\nu_{i}}^{Y}, i \in\{1, \ldots, s\}$. Рассмотрим случайные величины $\chi_{1}=\chi_{1}(n), \ldots, \chi_{s}=\chi_{s}(n)$, равные нулю на множестве $\Omega \backslash \widetilde{\Omega}$. На множестве $\widetilde{\Omega}$ величины $\chi_{1}, \ldots, \chi_{s}$ определим равенствами

$$
\begin{aligned}
& \chi_{1}=\sum_{k=1}^{s} I\left(\xi\left(\nu_{1}\right)=\cdots=\xi\left(\nu_{k}\right)\right)(k-1), \\
& \chi_{j}=\sum_{k=\sum_{l=1}^{j-1} \chi_{l}+j+1}^{s} I\left(\xi(1), \ldots, \xi(k) \in\left\{\xi(1), \ldots, \xi\left(\sum_{l=1}^{j-1} \chi_{l}+j\right)\right\}\right) k
\end{aligned}
$$

где $j \in\{2, \ldots, s\}, I(A)$ - индикатор события $A$.

Рассмотрим вероятностное пространство $(\widetilde{\Omega}, \widetilde{\mathscr{F}}, \widetilde{\mathbf{P}})$, где $\widetilde{\mathscr{F}}=\mathscr{F} \cap \widetilde{\Omega}$ и $\widetilde{\mathbf{P}}(A)=\mathbf{P}(A) / \mathbf{P}(\widetilde{\Omega})$ для $A \in \widetilde{\mathscr{F}}$. Нас будет интересовать поведение случайных величин $\chi_{1}^{*}=\chi_{1}^{*}(n), \ldots, \chi_{s}^{*}=\chi_{s}^{*}(n)$, заданных на пространстве $(\widetilde{\Omega}, \widetilde{\mathscr{F}}, \widetilde{\mathbf{P}})$ равенствами $\chi_{i}^{*}(\omega)=\chi_{i}(\omega)$, где $\omega \in \widetilde{\Omega}, i \in\{1, \ldots, s\}$.

Представим, что помимо $s$ частиц имеется еще счетное количество дополнительных частиц $\zeta_{s+1}=\zeta_{s+1}(n), \zeta_{s+2}=\zeta_{s+2}(n), \ldots$ Каждая из них совершает скачок независимо от остальных, при этом попадает с вероятностью $1 / u$ в любую точку с неактивной частицей. Иными словами, мы рассматриваем $\sigma$-алгебру $\widehat{\mathscr{F}}=\sigma\left(\widetilde{\mathscr{F}} \cup \sigma\left(\widehat{\chi}_{1}, \ldots, \widehat{\chi}_{u-1}\right)\right)$, где случайные величины $\widehat{\chi}_{i}=\widehat{\chi}_{i}(n), i \in\{1, \ldots, u-1\}$, определяются равенствами (8) и (9) для $j \in\{2, \ldots, u-1\}$. Очевидно, что соотношение $\widehat{\chi}_{j}=\chi_{j}^{*}$ выполнено на множестве $\left\{\chi_{1}^{*}+\cdots+\chi_{j}^{*}+j<s\right\}$ для всякого $j \in\{1, \ldots, s\}$. Пусть $\widehat{\mathbf{P}}$ - такая вероятностная мера, определенная на множествах из $\widehat{\mathscr{F}}$, что $\widehat{\mathbf{P}}(A)=\widetilde{\mathbf{P}}(A)$ для любого $A \in \widetilde{\mathscr{F}}$. Пусть случайная величина $\rho=\rho(n)$, заданная на вероятностном пространстве $(\widetilde{\Omega}, \widetilde{\mathscr{F}}, \widetilde{\mathbf{P}})$, равна количеству частиц, активированных в рассматриваемый момент времени $t$. Тогда $\widehat{\chi}_{1}+\cdots+\widehat{\chi}_{\rho}+\rho=s$. Кроме того, для всякого натурального $h<s$ имеем

$$
\widetilde{\mathbf{P}}\left(\chi_{1}^{*}+\cdots+\chi_{h}^{*}+h<s\right)=\widehat{\mathbf{P}}\left(\widehat{\chi}_{1}+\cdots+\widehat{\chi}_{h}+h<s\right) .
$$

Тогда $\widetilde{\mathbf{P}}(\rho>h)=\widehat{\mathbf{P}}\left(\widehat{\chi}_{1}+\cdots+\widehat{\chi}_{h}+h<s\right)$. Следовательно,

$$
\widetilde{\mathbf{P}}(\rho<h)=\widehat{\mathbf{P}}\left(\widehat{\chi}_{1}+\cdots+\widehat{\chi}_{h}+h \geqslant s\right) \leqslant \frac{\mathbf{D}_{\hat{\mathbf{P}}}\left(\widehat{\chi}_{1}+\cdots+\widehat{\chi}_{h}+h\right)}{\left(s-\mathbf{E}_{\hat{\mathbf{P}}}\left(\widehat{\chi}_{1}+\cdots+\widehat{\chi}_{h}+h\right)\right)^{2}} .
$$

Так как случайные величины $\widehat{\chi}_{1}, \ldots, \widehat{\chi}_{h}$ являются независимыми, то

$$
\mathbf{E}_{\hat{\mathbf{P}}}\left(\sum_{i=1}^{h} \widehat{\chi}_{i}+h\right)=u \ln \frac{u}{u-h}+O(1)
$$




$$
\mathbf{D}_{\hat{\mathbf{P}}}\left(\sum_{i=1}^{h} \widehat{\chi}_{i}\right)=u\left(\frac{h}{u-h}-\ln \frac{u}{u-h}\right)+O(1) .
$$

Поэтому при $\widetilde{h}=u\left(1-e^{-s / u}\right)$ для любого $\delta>0$ существует такая константа $\vartheta_{1}=\vartheta_{1}(\delta)>0$, что сразу для всех $n, s$ и $u$ таких, что $\widetilde{h}>n^{1 / 2-\alpha}$, имеет место неравенство

$$
\widetilde{\mathbf{P}}\left(|\rho-\widetilde{h}|>\widetilde{h}^{1 / 2+\delta}\right) \leqslant \vartheta_{1} s h^{-1-2 \delta} \leqslant \vartheta_{1} n^{-\delta(1 / 2-\alpha)} .
$$

Пусть известно количество активных частиц в момент $t$, а количество частиц, совершивших скачки в точки с неактивными частицами, неизвестно. Иными словами, пусть $w: \mathbf{N} \rightarrow \mathbf{N}$, причем $w(n)>n^{1 / 2-\alpha}$, $w(n)<u(n)$ для всех $n \in \mathbf{N}$. Для каждой частицы вероятность попасть в точку с неактивной частицей при условии $\left\{D_{n}(t)=u\right\}$ равна $p u n^{-1}$, а в другую точку $-p(n-u) n^{-1}$. Положим $\widetilde{\Omega}_{1}=\widetilde{\Omega}_{1}(n)=$ $\left\{D_{n}(t)=u, A_{n}(t)=w\right\}$. Из неравенства Берри-Эссеена (см. [13, с. 505507], [14, с. 157-158]) следует существование такой константы $\vartheta_{2}>0$, что для всех $n \in \mathbf{N}, n>e^{2 /(1-2 \alpha)}$

$$
\mathbf{P}\left(\left|-\Delta B_{n}(t)-w p(n-u) n^{-1}\right| \leqslant \sqrt{w} \ln \ln w \mid \widetilde{\Omega}_{1}\right) \geqslant 1-\vartheta_{2}(\ln w \ln \ln w)^{-1} .
$$

В силу (10) для $s$ частиц, совершивших скачки в точки с неактивными частицами, количество частиц, совершивших скачки в различные точки, с вероятностью $1-\vartheta_{1} n^{-\delta(1 / 2-\alpha)}$ находится в интервале $\left(\widetilde{h}-\widetilde{h}^{1 / 2+\delta}, \widetilde{h}+\right.$ $\left.\widetilde{h}^{1 / 2+\delta}\right)$. Иными словами, для любого $\delta>0$ найдется такое $\vartheta>0$, что сразу для всех $n \in \mathbf{N}$ (достаточно больших, чтобы можно было взять логарифм)

$$
\begin{gathered}
\mathbf{P}\left(\left|-\Delta D_{n}(t)-u\left(1-e^{-p w / n}\right)\right| \leqslant\left(u\left(1-e^{-p w / n}\right)\right)^{1 / 2+\delta} \mid \widetilde{\Omega}_{1}\right) \\
\geqslant 1-\vartheta(\ln n \ln \ln n)^{-1} .
\end{gathered}
$$

Из неравенств (11) и (12), очевидно, следует, что соотношения

$$
\begin{aligned}
& \left|\Delta\left(n-D_{n}(t)\right)-D_{n}(t)\left(1-e^{-p A_{n}(t) / n}\right)\right| \\
& \leqslant\left(D_{n}(t)\left(1-e^{-p A_{n}(t) / n}\right)\right)^{1 / 2+\delta} \\
& \left|\Delta A_{n}(t)+p\left(n-D_{n}(t)\right) A_{n}(t) n^{-1}-D_{n}(t)\left(1-e^{-p A_{n}(t) / n}\right)\right| \\
& \leqslant \sqrt{A_{n}(t)} \ln \ln A_{n}(t)+\left(D_{n}(t)\left(1-e^{-p A_{n}(t) / n}\right)\right)^{1 / 2+\delta}
\end{aligned}
$$

выполнены с вероятностью, превосходящей $1-\vartheta(\ln n \ln \ln n)^{-1}$. Теперь мы получили все необходимые утверждения о поведении частиц. Обратимся к оценке интересующих нас случайных величин. Вначале (в п. 3.3) по индукции оценим количество активных частиц и неактивных частиц, а затем (в п. 3.4$)$ - величину $X_{n}$. 
3.3. Оценка количества активных частиц. Пусть $\delta$ - произвольное положительное число. Для всех $i \in\left[t_{\alpha}(n), \infty\right) \cap \mathbf{N}$ положим

$$
\begin{gathered}
z_{n}^{(1)}\left(t_{\alpha}(n)\right)=z_{n}^{(2)}\left(t_{\alpha}(n)\right)=n^{1 / 2-\alpha}, \quad r_{n}^{(1)}\left(t_{\alpha}(n)\right)=r_{n}^{(2)}\left(t_{\alpha}(n)\right)=n^{1 / 4+\delta} \\
z_{n}^{(1)}(i+1)=z_{n}^{(1)}(i)+\left(n-z_{n}^{(1)}(i)\right)\left(1-\exp \left(-p z_{n}^{(2)}(i) n^{-1}\right)\right) \\
z_{n}^{(2)}(i+1)= \\
z_{n}^{(2)}(i)\left(1-p z_{n}^{(1)}(i) n^{-1}\right) \\
+\left(n-z_{n}^{(1)}(i)\right)\left(1-\exp \left(-p z_{n}^{(2)}(i) n^{-1}\right)\right) .
\end{gathered}
$$

Прежде чем получить оценки на количество активных и неактивных частиц, изучим поведение функций $z_{n}^{(1)}(i)$ и $z_{n}^{(2)}(i)$.

Лемма 3. Пусть $C_{1} \in(1 / 2,18 / 35)$ - некоторая константа, $p_{0}=$ $(4-p) /(8-p)$. Пусть, кроме того, для некоторого $i_{0}(n)$ выполнено неравенство $z_{n}^{(1)}\left(i_{0}(n)\right) \leqslant C_{1} n$ для всех $n$. Тогда

$$
\exists H>1 \forall n \in \mathbf{N} \forall i \in\left[t_{\alpha}(n), i_{0}\right] \quad \frac{z_{n}^{(1)}(i+1)}{z_{n}^{(1)}(i)} \geqslant H .
$$

Итак, пусть $z_{n}^{(1)}\left(i_{0}(n)\right) \leqslant C_{1} n, i \in\left\{t_{\alpha}(n), t_{\alpha}(n)+1, \ldots, i_{0}(n)\right\}$. Заметим, что $z_{n}^{(2)}(i) \leqslant n / 2$, так как функция $z_{n}^{(2)}(i)$ убывает при всех $i$ таких, что $z_{n}^{(1)}(i) \geqslant n / 2$, а $z_{n}^{(2)}(i) \leqslant z_{n}^{(1)}(i)$. Поэтому справедливо неравенство

$\left(n-z_{n}^{(1)}(i)\right)\left(1-\exp \left(-p z_{n}^{(2)}(i) n^{-1}\right)\right) \geqslant p\left(\frac{1-p}{4}\right)\left(z_{n}^{(2)}(i)-z_{n}^{(1)}(i) z_{n}^{(2)}(i) n^{-1}\right)$.

Пусть после $i_{1}$-го шага $z_{n}^{(2)}(i)$ уменьшается, тогда в силу полученного неравенства имеем $p(1-p / 4)<p(2-p / 4) z_{n}^{(1)}\left(i_{1}\right) n^{-1}, z_{n}^{(1)}\left(i_{1}\right)>n p_{0}$. Докажем, что

$$
z_{n}^{(2)}\left(i_{1}\right)>\frac{n p_{0}}{5} .
$$

Если $z_{n}^{(1)}(i)<n p_{0} / 2$, то, так как функция $8(8-p) /(6-p)^{2}$ возрастает на отрезке $[0,1]$, имеем

$$
\begin{aligned}
\frac{z_{n}^{(1)}(i+1)-z_{n}^{(1)}(i)}{z_{n}^{(2)}(i+1)-z_{n}^{(2)}(i)} & <\frac{p\left(1-z_{n}^{(1)}(i) n^{-1}\right)}{p(1-p / 4)\left(1-z_{n}^{(1)}(i) n^{-1}\right)-p z_{n}^{(1)}(i) n^{-1}} \\
& <\frac{8(8-p)}{(6-p)^{2}}<\frac{56}{25}, \\
& z_{n}^{(2)}\left(i_{1}\right)>\frac{25}{56} \frac{n p_{0}}{2}>\frac{n p_{0}}{5} .
\end{aligned}
$$

При этом неравенства $z_{n}^{(2)}(i+1)>z_{n}^{(2)}(i)\left(1+p(4-p) / 4-p(8-p) C_{1} / 4\right)>$ $z_{n}^{(2)}(i) \rho$, где $\rho=1-p(2+p) / 10$, справедливы для любого $i>i_{1}$, так как $C_{1}<3 / 5$. Выберем $i_{2}(n)$ такое, что

$$
z_{n}^{(1)}\left(i_{2}\right) \geqslant n\left(p_{0}+\frac{p(4-p) p_{0}}{20} \cdot 2\left(1-\rho^{i_{2}-i_{1}}\right)(5(1-\rho))^{-1}\right)>C_{1} n .
$$


Существование упомянутого $i_{2}$ обеспечивается неравенством (16), а также цепочкой соотношений

$$
p_{0}+\frac{p(4-p) p_{0}}{50}(1-\rho)^{-1}=p_{0}\left(1+(4-p)(5(2+p))^{-1}\right) \geqslant \frac{18}{35},
$$

где последнее неравенство выполнено, так как функция, фигурирующая в левой части (18), убывает на отрезке $[0,1]$. Подходящее значение $i_{2}$ найдем из следующей оценки:

$$
\rho^{i_{2}-i_{1}}<(4 p+14)(4-p)^{-1}-5 C_{1}(2+p)(8-p)(4-p)^{-2} .
$$

Положим

$i_{2}=i_{1}+\left[\ln ^{-1} \rho \ln \left((4 p+14)(2(4-p))^{-1}-5 C_{1}(2+p)(8-p)(2(4-p))^{-2}\right)\right]$.

Тогда в силу (17)

$z_{n}^{(2)}\left(i_{2}\right)>z_{n}^{(2)}\left(i_{1}\right) \rho^{i_{2}-i_{1}-1}>n(5(8-p))^{-1}\left(4 p+14-5 C_{1}(2+p)(8-p)(4-p)^{-1}\right)$.

Окончательно заметим, что для всех $i \leqslant i_{2}$

$$
z_{n}^{(1)}(i+1) \geqslant z_{n}^{(1)}(i)+p\left(1-\frac{p}{4}\right)\left(z_{n}^{(2)}(i)-z_{n}^{(1)}(i) z_{n}^{(2)}(i) n^{-1}\right) \geqslant z_{n}^{(1)}(i)+C_{2} n,
$$

где

$C_{2}=\left(1-C_{1}\right) p\left(1-\frac{p}{4}\right)(5(8-p))^{-1}\left(4 p+14-5 C_{1}(2+p)(8-p)(4-p)^{-1}\right)$.

Таким образом, лемма 3 доказана.

Легко заметить, что при $i>C_{1} n$ функция $z_{n}^{(2)}(i)$ экспоненциально убывает по $i$. Поэтому для любого положительного $\kappa<1$ найдется $i_{\kappa}=i_{\kappa}(n)=O(n)$ такое, что $z_{n}^{(2)}\left(i_{\kappa}\right)=O\left(n^{\kappa}\right), z_{n}^{(1)}\left(i_{\kappa}\right) \geqslant C_{1} n$. При этом, если $\kappa_{m} \rightarrow 1, m \rightarrow \infty$ и для некоторого $i=i(n)$ справедливы неравенства $z_{n}^{(2)}(i) \geqslant c n, z_{n}^{(1)}(i) \leqslant n / 2$ при всех $n \in \mathbf{N}$, то $\left(i_{\kappa_{m}}-i\right) / \ln n \rightarrow 0(m \rightarrow \infty)$ при каждом $n \in \mathbf{N}$.

Лемма 4. Для любых $\lambda_{1}>\lambda>0$ найдутся такие числа $\kappa, \kappa_{1} \in$ $(3 / 4,1), \kappa_{1}<\kappa$, натуральные числа $i_{\kappa}=i_{\kappa}(n), i_{\kappa_{1}}=i_{\kappa_{1}}(n), i_{3 / 4}=i_{3 / 4}(n)$, $i_{\kappa} \leqslant i_{\kappa_{1}} \leqslant i_{3 / 4}$, а также такое множество $\Omega_{n} \subset \Omega$, что $\mathbf{P}\left(\Omega_{n}\right) \geqslant 1-$ $\vartheta \ln ^{-1} \ln n$. Кроме того, для каждого $i \in\left\{t_{\alpha}(n), t_{\alpha}(n)+1, \ldots, i_{3 / 4}-1\right\} u$ всех $\omega \in \Omega_{n}^{1}$ выполнены неравенства

$$
\left|n-D_{n}(i)-z_{n}^{(1)}(i)\right| \leqslant r_{n}^{(1)}(i), \quad\left|A_{n}(i)-z_{n}^{(2)}(i)\right| \leqslant r_{n}^{(2)}(i),
$$

где $r_{n}^{(1)}, r_{n}^{(2)}: \mathbf{N} \rightarrow \mathbf{R}_{+}-$бункиии, для которых справедливь следуюшие свойства. 
I. Имеют место соотношения $z_{n}^{(2)}\left(i_{\kappa}\right) \leqslant n^{\kappa}, z_{n}^{(1)}\left(i_{\kappa}\right)<n / 2 u$ $r_{n}^{(1)}\left(i_{\kappa}\right)\left(r_{n}^{(1)}\left(t_{\alpha}(n)\right)\right)^{-1}=r_{n}^{(2)}\left(i_{\kappa}\right)\left(r_{n}^{(2)}\left(t_{\alpha}(n)\right)\right)^{-1} \leqslant n^{\lambda} z_{n}^{(1)}\left(i_{\kappa}\right)\left(z_{n}^{(1)}\left(t_{\alpha}(n)\right)\right)^{-1}$.

II. Справедливьь неравенства $z_{n}^{(2)}\left(i_{\kappa_{1}}\right) \leqslant n^{\kappa_{1}}, z_{n}^{(1)}\left(i_{\kappa_{1}}\right) \geqslant C_{1} n u$ $r_{n}^{(1)}\left(i_{\kappa_{1}}\right) \leqslant n^{\lambda_{1}} r_{n}\left(i_{\kappa}\right)$.

III. Для любого $i \in\left\{i_{\kappa_{1}}, i_{\kappa_{1}}+1, \ldots, i_{3 / 4}-1\right\}$ найдется такое число $c>0$, umo

$$
r_{n}^{(1)}(i+1) \leqslant r_{n}^{(1)}(i)\left(1+c n^{\kappa_{1}-\kappa}\right) .
$$

Д о к а з а т е л ь с т в о. Из свойств функций $z_{n}^{(1)}(i), z_{n}^{(2)}(i)$ вытекает, что найдется такое натуральное число $i_{3 / 4}=i_{3 / 4}(n)$, что $z_{n}^{(2)}\left(i_{3 / 4}\right) \leqslant$ $n^{3 / 4}, z_{n}^{(1)}\left(i_{3 / 4}\right) \geqslant C_{1} n$. Возьмем в качестве множества $\widetilde{\Omega}_{n}\left(t_{\alpha}(n)\right)$ множество из формулировки леммы 2 , мера которого стремится к 1 . Пусть $\kappa$ - такое число, что $z_{n}^{(2)}\left(i_{\kappa}\right) \leqslant n^{\kappa}, z_{n}^{(1)}\left(i_{\kappa}\right)<n / 2$. Если неравенства (19) выполнены для шага с номером $i \in\left\{t_{\alpha}(n), t_{\alpha}(n)+1, \ldots, i_{\kappa}-1\right\}$, то согласно п. 3.2 найдется множество $\Omega_{n}(i+1) \subset \Omega_{n}(i)$ такое, что

$$
\mathbf{P}\left(\Omega_{n}(i+1)\right) \geqslant\left(1-\vartheta(\ln \ln n \ln n)^{-1}\right) \mathbf{P}\left(\Omega_{n}(i)\right)
$$

и для каждого $\omega \in \Omega_{n}(i+1)$ в силу $(14)$

$$
\begin{aligned}
& \left|A_{n}(i+1)-z_{n}^{(2)}(i+1)\right| \\
& \leqslant \mid-p z_{n}^{(2)}(i) r_{n}(i) n^{-1}+\left(n-z_{n}^{(1)}(i)\right) e^{-p z_{n}^{(2)}(i) / n}\left(1-e^{-p r_{n}(i) / n}\right) \\
& \quad+r_{n}(i)\left(1-p\left(z_{n}^{(1)}(i)+r_{n}(i)\right) n^{-1}\right)-r_{n}(i)\left(1-e^{-p\left(z_{n}^{(2)}(i)+r_{n}(i)\right) / n}\right) \mid \\
& \quad+\left(p A_{n}(i)\right)^{1 / 2+\delta} \\
& \leqslant \\
& \quad\left(n-z_{n}^{(1)}(i)\right)\left(1-e^{-p r_{n}(i) / n}\right) e^{-p z_{n}^{(2)}(i) / n}+r_{n}(i)\left(1+n^{-(\delta / 2+\alpha / 2+\alpha \delta)}\right) .
\end{aligned}
$$

Аналогичным образом, в силу (13)

$$
\begin{aligned}
& \left|n-D_{n}(i+1)-z_{n}^{(2)}(i+1)\right| \\
& \quad \leqslant\left(n-z_{n}^{(1)}(i)\right)\left(1-e^{-p r_{n}(i) / n}\right) e^{-p z_{n}^{(2)}(i) / n}+r_{n}(i)\left(1+n^{-(\delta / 2+\alpha / 2+\alpha \delta)}\right) .
\end{aligned}
$$

Поэтому мы можем считать, что $r_{n}^{(1)}(i+1)=r_{n}^{(2)}(i+1)=r_{n}(i+1)$,

$$
\begin{aligned}
r_{n}(i+1)= & \left(n-z_{n}^{(1)}(i)\right)\left(1-e^{-p r_{n}(i) / n}\right) e^{-p z_{n}^{(2)}(i) / n} \\
& +r_{n}(i)\left(1+n^{-(\delta / 2+\alpha / 2+\alpha \delta)}\right) .
\end{aligned}
$$

Используя это равенство, легко получить, что для любого $\varepsilon>0$ найдется такое $c>0$, что если $z_{n}^{(1)}(i)<$ cn для всех $n$, то $r_{n}(i+1)\left(r_{n}(i)\right)^{-1}<$ $(1+\varepsilon) z_{n}^{(1)}(i+1)\left(z_{n}^{(1)}(i)\right)^{-1}$. Из этого, в свою очередь, следует, что для 
любого $\tilde{\lambda}>0$ существует такое $c>0$, что если $z_{n}^{(1)}(i)<c n$ для всех $n$, то $r_{n}(i)\left(r_{n}\left(t_{\alpha}(n)\right)\right)^{-1}<n^{\widetilde{\lambda}} z_{n}^{(1)}(i)\left(z_{n}^{(1)}\left(t_{\alpha}(n)\right)\right)^{-1}$. Заметим, наконец, что $r_{n}(i+1) \leqslant(1+p) r_{n}^{(1)}(i)$. Поэтому, если $\kappa_{m} \rightarrow 1-0, m \rightarrow \infty$, то

$$
r_{n}\left(i_{\kappa_{m}}\right)\left(r_{n}\left(t_{\alpha}(n)\right)\right)^{-1}<n^{\widetilde{\lambda}+\lambda_{m}} z_{n}^{(1)}\left(i_{\kappa_{m}}\right)\left(z_{n}^{(1)}\left(t_{\alpha}(n)\right)\right)^{-1}
$$

где $\lambda_{m} \rightarrow+0, m \rightarrow \infty$. Свойство I доказано.

Согласно (13) и (14), для каждого $i \in\left\{i_{\kappa}, i_{\kappa}+1, \ldots, i_{3 / 4}-1\right\}$ можно выбрать множество $\Omega_{n}(i+1) \subset \Omega_{n}(i)$ такое, что выполнено неравенство $(21)$ и для всех $\omega \in \Omega_{n}(i+1)$ справедливы неравенства

$$
\left|A_{n}(i+1)-z_{n}^{(2)}(i+1)\right| \leqslant r_{n}^{(1)}(i)+p r_{n}^{(2)}(i)\left(1-z_{n}^{(1)}(i) n^{-1}\right)+\left(z_{n}^{(2)}(i)\right)^{1 / 2+\delta} .
$$

Поэтому мы можем считать, что

$$
\begin{aligned}
& r_{n}^{(1)}(i+1)=r_{n}^{(1)}(i)+p r_{n}^{(2)}(i)\left(1-z_{n}^{(1)}(i) n^{-1}\right)+\left(z_{n}^{(2)}(i)\right)^{1 / 2+\delta}, \\
& r_{n}^{(2)}(i+1)=r_{n}^{(2)}(i)\left(1+p-2 p z_{n}^{(1)}(i) n^{-1}\right)+\left(2 z_{n}^{(2)}(i)\right)^{1 / 2+\delta} .
\end{aligned}
$$

Следовательно, $r_{n}^{(1)}(i+1) \leqslant(1+p) r_{n}^{(1)}(i)$, т.е. в силу свойств $z_{n}^{(1)}(i), z_{n}^{(2)}(i)$ найдется нужное число $\kappa_{1}$ (число $\kappa$ при этом необходимо выбрать достаточно близким к 1). Свойство II доказано.

Для доказательства свойства III заметим, что существует такое число $C>0$, что для каждого $i \in\left\{i_{\kappa}, i_{\kappa}+1, \ldots, i_{3 / 4}-1\right\}$ справедливы неравенства

$$
\begin{aligned}
z_{n}^{(2)}(i+1)\left(z_{n}^{(2)}(i)\right)^{-1} & \leqslant r_{n}^{(2)}(i+1)\left(r_{n}^{(2)}(i)\right)^{-1} \\
& \leqslant z_{n}^{(2)}(i+1)\left(z_{n}^{(2)}(i)\right)^{-1}+C\left(z_{n}^{(2)}(i)\right)^{\delta-1 / 4} .
\end{aligned}
$$

Следовательно, если $z_{n}^{(2)}(i+1) \leqslant n^{\kappa_{1}}$, а $z_{n}^{(1)}(i+1) \geqslant C_{1} n$, то для некоторого $\widetilde{c}>0$

$$
r_{n}^{(2)}(i+1) \leqslant \widetilde{c} n^{\kappa_{1}-\kappa} r_{n}^{(2)}\left(i_{\kappa}\right)=\widetilde{c} n^{\kappa_{1}-\kappa} r_{n}^{(1)}\left(i_{\kappa}\right) \leqslant \widetilde{c} n^{\kappa_{1}-\kappa} r_{n}^{(1)}(i+1) .
$$

Окончательно получаем

$$
\begin{aligned}
r_{n}^{(1)}(i+1) & =r_{n}^{(1)}(i)+p r_{n}^{(2)}(i)\left(1-z_{n}^{(1)}(i) n^{-1}\right)+\left(z_{n}^{(2)}(i)\right)^{1 / 2+\delta} \\
& \leqslant r_{n}^{(1)}(i)\left(1+c n^{\kappa_{1}-\kappa}\right)
\end{aligned}
$$

для некоторого $c>0$. В качестве множества $\Omega_{n}$ возьмем пресечение множеств $\Omega_{n}(i)$ по $i \in\left\{t_{\alpha}(n), t_{\alpha}(n)+1, \ldots, i_{3 / 4}\right\}$. Лемма 4 доказана. 
3.4. Оценка количества умерших частиц. Из леммы 4 следует, что для любого $\varepsilon>0$ существует такое число $i_{3 / 4} \in \mathbf{N}$, что

$$
\begin{aligned}
\mathbf{P}\left(\left(A_{n}\left(i_{3 / 4}\right) \leqslant n^{3 / 4}\right)\right. & \left.\wedge\left(\left|n-D_{n}\left(i_{3 / 4}\right)-z_{n}^{(1)}\left(i_{3 / 4}\right)\right| \leqslant n^{3 / 4+\varepsilon}\right)\right) \\
& \geqslant 1-\vartheta \ln ^{-1} \ln n
\end{aligned}
$$

при этом $z_{n}^{(1)}\left(i_{3 / 4}\right) \geqslant C_{1} n$.

Пусть $\widetilde{\sigma}_{n}$ - такой момент времени, что $n-D_{n}\left(\widetilde{\sigma}_{n}\right) \geqslant C_{1} n, A_{n}\left(\widetilde{\sigma}_{n}\right) \leqslant$ $\ln ^{2} n$ и $A_{n}\left(\widetilde{\sigma}_{n}+1\right)>\ln ^{2} n$. Пусть, кроме того $\widehat{\sigma}_{n}=\max \left\{\widetilde{\sigma}_{n}, i_{3 / 4}\right\}$. Опираясь на неравенство Чернова и неравенство $C_{1}>1 / 2$, легко показать, что найдется число $\widetilde{C}>0$, для которого

$$
\lim _{n \rightarrow \infty} \mathbf{P}\left(\left(\widehat{\sigma}_{n} \leqslant \widetilde{C} \ln n\right) \wedge\left(D_{n}\left(i_{3 / 4}\right)-D_{n}\left(\widehat{\sigma}_{n}\right) \leqslant \widetilde{C} n^{3 / 4}\right)\right)=1 .
$$

Осталось доказать, что для любого $\delta>0$ и некоторого числа $C>0$

$$
\lim _{n \rightarrow \infty} \mathbf{P}\left(\left(\sigma_{n}-\widehat{\sigma}_{n} \leqslant C \ln \ln n\right) \wedge\left(D_{n}\left(\widehat{\sigma}_{n}\right)-D_{n}\left(\sigma_{n}\right) \leqslant C n^{\delta}\right)\right)=1 .
$$

Рассмотрим ветвящийся процесс $T_{t}, t \in \mathbf{N}$ (см., например, [15, с. 125-126]): $T_{1}=\tau_{1}^{1}, T_{t}=\tau_{1}^{t}+\cdots+\tau_{T_{t-1}}^{t}$, где $\tau_{i}^{j} \in\{0,1,2\}$, $i, j \in\{1,2, \ldots\}$ - независимые одинаково распределенные величины, $\mathbf{P}\left(\tau_{1}^{1}=0\right)=p C_{1}, \mathbf{P}\left(\tau_{1}^{1}=1\right)=1-p, \mathbf{P}\left(\tau_{1}^{1}=2\right)=p\left(1-C_{1}\right)$. Докажем по индукции, что вероятность вырождения такого процесса в момент $t$ не меньше, чем $1-\left(1+p-2 p C_{1}\right)^{t}$. Для момента 1 это очевидно: $p C_{1}>2 p C_{1}-p$. Производящая функция случайной величины $\tau_{1}^{1}$ имеет вид $P(x)=p C_{1}+(1-p) x+p\left(1-C_{1}\right) x^{2}$. Поэтому, если в момент $t$ вероятность вырождения не меньше этой величины, то в момент $t+1$ вероятность вырождения не превосходит

$$
P\left(1-\left(1+p-2 p C_{1}\right)^{t}\right)<1-\left(1+p-2 p C_{1}\right)^{t+1} .
$$

Но вероятность смерти одной частицы в любой момент, больший $i_{3 / 4}$, превышает $p C_{1}$, поэтому за время $t$ с вероятностью, превосходящей $1-$ $\left(1+p-2 p C_{1}\right)^{t}$, погибнут все потомки этой частицы. Следовательно, $\ln ^{2} n$ частиц умрут за время $t$ с вероятностью большей, чем $1-\ln ^{2} n(1+$ $\left.p-2 p C_{1}\right)^{t}$. Так как $C_{1}>1 / 2$, то найдется такое число $C>0$, что при $t=[C \ln n \ln n]$ выполнено соотношение $1-\ln ^{2} n\left(1+p-2 p C_{1}\right)^{t}=1+o(1)$. Кроме того,

$$
D_{n}\left(\sigma_{n}\right) \leqslant D_{n}\left(\widehat{\sigma}_{n}+t\right)+\left(\ln ^{2} n\right) \cdot 2^{t} \leqslant D_{n}\left(\widehat{\sigma}_{n}+t\right)+C n^{\delta}
$$

с вероятностью, стремящейся к 1 . Таким образом, для любого $\varepsilon>0$

$$
\lim _{n \rightarrow \infty} \mathbf{P}\left(\left|X_{n}-z_{n}^{(1)}\left(i_{3 / 4}\right)\right| \leqslant n^{3 / 4+\varepsilon}\right)=1 .
$$


Можно показать, что это равенство сохранится, если $z_{n}^{(1)}\left(i_{3 / 4}\right)$ заменить на $\mathbf{E} X_{n}$. Подробное доказательство этого факта мы здесь не приводим, поскольку оно достаточно объемное и при этом не представляет отдельного интереса (подобное рассуждение мы уже проделывали в п. 3.1). Суть заключается в следующем. Можно показать, что с вероятностью $1+O\left(n^{\psi-1 / 4}\right)$, где $\psi>\alpha$, найдется момент времени, в который будет активировано $n^{1 / 2-\alpha}+r$ частиц, где $|r| \leqslant n^{1 / 4-\alpha / 2+\delta}$. Далее оценки из п. 3.2 можно улучшить таким образом, чтобы неравенства (21) приобрели вид $\mathbf{P}\left(\Omega_{n}(i+1)\right) \geqslant\left(1-\vartheta\left(n^{1 / 4-\psi} \ln n\right)^{-1}\right) \mathbf{P}\left(\Omega_{n}(i)\right)$. Тогда $\left|X_{n}-z_{n}^{(1)}\left(i_{3 / 4}\right)\right| \leqslant n^{3 / 4+\varepsilon}$ с вероятностью $1+O\left(n^{\psi-1 / 4}\right)$. Поэтому при достаточно больших $n$ имеем

$$
\left(z_{n}^{(1)}\left(i_{3 / 4}\right)-n^{3 / 4+\varepsilon}\right)\left(1-n^{\psi-1 / 4}\right) \leqslant \mathbf{E} X_{n} \leqslant z_{n}^{(1)}\left(i_{3 / 4}\right)+n^{3 / 4+\varepsilon}+n \cdot n^{\psi-1 / 4} .
$$

Следовательно, для любого $\psi>0$ при достаточно больших $n$

$$
z_{n}^{(1)}\left(i_{3 / 4}\right)-n^{3 / 4+\psi} \leqslant \mathbf{E} X_{n} \leqslant z_{n}^{(1)}\left(i_{3 / 4}\right)+n^{3 / 4+\psi} .
$$

Таким образом, для любого $\varepsilon>0$ имеем $\lim _{n \rightarrow \infty} \mathbf{P}\left(\left|X_{n}-\mathbf{E} X_{n}\right| \leqslant\right.$ $\left.n^{3 / 4+\varepsilon}\right)=1$.

3.5. Оптимальность показателя степени. Пусть $R_{n}^{(1)}\left(t_{\alpha}(n)\right)=$ $R_{n}^{(2)}\left(t_{\alpha}(n)\right)=n^{1 / 4-\alpha / 2-\delta}$, где $\delta>0-$ произвольное число.

Лемма 5. Для любых $\mu>0, \lambda>0, \varepsilon \in(0,3 / 4), \varrho \in(0,1)$ найдутся такие натуральнье числа $i_{\varrho}=i_{\varrho}(n), j_{\varrho}=j_{\varrho}(n), i_{3 / 4-\varepsilon}=i_{3 / 4-\varepsilon}(n), a$ также такое множество $\widetilde{\Omega}_{n}$, что при достаточно больших $n$ выполнено неравенство $\mathbf{P}\left(\widetilde{\Omega}_{n}\right)>1 / 2-\mu$. Кроме того, для всех $\omega \in \widetilde{\Omega}_{n} u$ $i \in\left\{t_{\alpha}(n), t_{\alpha}(n)+1, \ldots, i_{3 / 4-\varepsilon}\right\}$ справедливы неравенства

$$
\left|n-D_{n}(i)-z_{n}^{(1)}(i)\right| \geqslant R_{n}^{(1)}(i), \quad\left|A_{n}(i)-z_{n}^{(2)}(i)\right| \geqslant R_{n}^{(2)}(i),
$$

причем

$$
\begin{gathered}
z_{n}^{(2)}\left(j_{\varrho}\right) \geqslant n^{\varrho}, \quad \frac{n}{2}>z_{n}^{(1)}\left(j_{\varrho}\right) \geqslant n^{\varrho}, \quad z_{n}^{(2)}\left(i_{\varrho}\right) \leqslant n^{\varrho}, \\
z_{n}^{(1)}\left(i_{\varrho}\right) \geqslant C_{1} n, \quad z_{n}^{(2)}\left(i_{3 / 4-\varepsilon}\right) \leqslant n^{3 / 4-\varepsilon}, \quad z_{n}^{(1)}\left(i_{3 / 4-\varepsilon}\right) \geqslant C_{1} n,
\end{gathered}
$$

а величина $R_{n}^{(1)}(i)$ обладает следуюшими свойствами.

IV. Вьполнено неравенство $R_{n}^{(1)}\left(j_{\varrho}\right)\left(R_{n}^{(1)}\left(t_{\alpha}(n)\right)\right)^{-1} \geqslant n^{-\lambda} z_{n}^{(1)}\left(j_{\varrho}\right) \times$ $\left(z_{n}^{(1)}\left(t_{\alpha}(n)\right)\right)^{-1}$.

V. Найдется такое число $c>0$, ито $R_{n}^{(1)}(i+1) \geqslant c R_{n}^{(1)}(i)$ nрu $i \in\left\{j_{\varrho}, j_{\varrho}+1, \ldots, i_{\varrho}\right\}$.

VI. Для любого $\epsilon>0$ при достаточно больших $n u i \in\left\{i_{\varrho}, i_{\varrho}+\right.$ $\left.1, \ldots, i_{3 / 4-\varepsilon}\right\}$ имеет место неравенство $R_{n}^{(1)}(i+1) \geqslant(1-\epsilon) R_{n}^{(1)}(i)$. 
Д о к а з а т е л ь с т в о. Справедливость неравенств (22) мы установим по индукции. Базой индукции служит утверждение леммы 2. Возьмем в качестве $\widetilde{\Omega}_{n}\left(t_{\alpha}(n)\right)$ множество из формулировки леммы 2 , мера которого стремится к 1 . Пусть $\epsilon>0, \widetilde{\varrho} \in(0,1)$ - произвольные числа. Найдутся такие числа $i_{\varrho}, j_{\varrho}, i_{3 / 4-\varepsilon}$, что

$$
\begin{aligned}
& n^{(1+\varrho) / 2} \geqslant z_{n}^{(2)}\left(j_{\varrho}\right) \geqslant n^{\varrho}, \quad \frac{n}{2}>z_{n}^{(1)}\left(j_{\varrho}\right) \geqslant n^{\varrho}, \quad z_{n}^{(2)}\left(i_{\varrho}\right) \leqslant n^{\varrho}, \\
& z_{n}^{(1)}\left(i_{\varrho}\right) \geqslant C_{1} n, \quad z_{n}^{(2)}\left(i_{3 / 4-\varepsilon}\right) \leqslant n^{3 / 4-\varepsilon}, \quad z_{n}^{(1)}\left(i_{3 / 4-\varepsilon}\right) \geqslant C_{1} n .
\end{aligned}
$$

Тогда существует такое число $N_{0} \in \mathbf{N}$, что если соотношения (22) выполнены для некоторого шага с номером $i \in\left\{t_{\alpha}(n), t_{\alpha}(n)+1, \ldots, j_{\varrho}-1\right\}$, то в силу неравенства $(13)$ найдется множество $\widetilde{\Omega}_{n}(i+1) \subset \widetilde{\Omega}_{n}(i)$ такое, что

$$
\mathbf{P}\left(\widetilde{\Omega}_{n}(i+1)\right) \geqslant\left(1-\vartheta(\ln \ln n \ln n)^{-1}\right) \mathbf{P}\left(\widetilde{\Omega}_{n}(i)\right)
$$

и при $n>N_{0}$ неравенство $\left|n-D_{n}(i+1)-z_{n}^{(1)}(i+1)\right| \geqslant(1-\epsilon)(1+p) R_{n}^{(1)}(i)$ справедливо для каждого $\omega \in \widetilde{\Omega}_{n}(i+1)$. Поэтому мы можем считать, что $R_{n}^{(1)}(i+1)=(1-\epsilon)(1+p) R_{n}^{(1)}$, а в качестве множества $\widetilde{\Omega}_{n}$ взять $\bigcap_{i=t_{\alpha}(n)}^{\varrho} \widetilde{\Omega}_{n}(i+1)$. С другой стороны, $z_{n}^{(1)}(i+1) \leqslant z_{n}^{(1)}(i)(1+p)$. Следовательно, $R_{n}^{(1)}(i+1)\left(R_{n}^{(1)}(i)\right)^{-1} \geqslant(1-\epsilon) z_{n}^{(1)}(i+1)\left(z_{n}^{(1)}(i)\right)^{-1}$. Тогда для некоторого $h>0$, не зависящего от $\epsilon$,

$$
R_{n}^{(1)}\left(j_{\varrho}\right)\left(R_{n}^{(1)}\left(t_{\alpha}(n)\right)\right)^{-1} \geqslant(1-\epsilon)^{h \ln n} z_{n}^{(1)}\left(j_{\varrho}\right)\left(z_{n}^{(1)}\left(t_{\alpha}(n)\right)\right)^{-1} .
$$

Если в качестве $\epsilon$ взять $1-e^{-\lambda / h}$, то мы получим свойство IV.

Существует такое число $N_{1} \in \mathbf{N}$, что если соотношения (22) выполнены для шага с номером $i \in\left\{j_{\varrho}, j_{\varrho}+1, \ldots, i_{\varrho}-1\right\}$, то в силу (13) найдется такое множество $\widetilde{\Omega}_{n}(i+1) \subset \widetilde{\Omega}_{n}(i)$, что неравенства $(23)$ и

$$
\begin{aligned}
&(1-\epsilon)^{-1}\left|n-D_{n}(i+1)-z_{n}^{(1)}(i+1)\right| \\
& \geqslant R_{n}^{(1)}(i)\left(1-p\left(z_{n}^{(2)}(i)+R_{n}^{(2)}(i)\right) n^{-1}\right) \\
&+R_{n}^{(1)}(i)\left(n-z_{n}^{(1)}(i)\right)\left(1-p z_{n}^{(2)}(i) n^{-1}\right) p R_{n}^{(2)}(i) n^{-1} \\
& \geqslant(1-\epsilon)\left(1-\frac{p}{2}\right) R_{n}^{(1)}(i)
\end{aligned}
$$

справедливы при $n>N_{1}$ и для каждого $\omega \in \widetilde{\Omega}_{n}(i+1)$. Поэтому мы можем считать, что $R_{n}^{(1)}(i+1)=(1-\epsilon)^{2}(1-p / 2) R_{n}^{(1)}(i)$. Свойство V доказано.

Рассмотрим, наконец, отрезок $\left[i_{\varrho}, i_{3 / 4-\varepsilon}\right]$. Существует такое число $N_{2} \in \mathbf{N}$, что если соотношения (22) выполнены для шага с номером 
$i \in\left\{i_{\varrho}, i_{\varrho}+1, \ldots, i_{3 / 4-\varepsilon}\right\}$, то согласно п. 3.2 найдется такое множество $\widetilde{\Omega}_{n}(i+1) \subset \widetilde{\Omega}_{n}(i)$, что неравенства $(23)$ и

$$
\begin{aligned}
\left|n-D_{n}(i+1)-z_{n}^{(1)}(i+1)\right| & \geqslant(1-\epsilon)\left(R_{n}^{(1)}(i)+\left(n-z_{n}^{(1)}(i)\right) p R_{n}^{(2)}(i) n^{-1}\right) \\
& \geqslant(1-\epsilon) R_{n}^{(1)}(i)
\end{aligned}
$$

справедливы при $n>N_{2}$ и для каждого $\omega \in \widetilde{\Omega}_{n}(i+1)$. Свойство VI доказано.

Из леммы 5 следует, что $\left|n-D_{n}\left(i_{3 / 4-\varepsilon}\right)-z_{n}^{(1)}\left(i_{3 / 4-\varepsilon}\right)\right| \geqslant R_{n}^{(1)}\left(i_{3 / 4-\varepsilon}\right)$ с вероятностью, стремящейся к 1 при $n \rightarrow \infty$. Кроме того, для некоторого $h>0$ и любых $\epsilon>0, \lambda>0$ и последовательности $\left\{\mu_{m}\right\}_{m \in \mathbf{N}}, \mu_{m}>0$, $\mu_{m} \rightarrow 0$, найдется последовательность $\left\{\varrho_{m}\right\}_{m \in \mathbf{N}}, \varrho_{m} \in(0,1), \varrho_{m} \rightarrow 1$ такая, что выполнено неравенство

$$
R_{n}^{(1)}\left(i_{3 / 4-\varepsilon}\right) \geqslant n^{1 / 4-\alpha / 2-\delta} n^{-\lambda} n^{\varrho_{m}} n^{\alpha-1 / 2} c^{\mu_{m} \ln n}(1-\epsilon)^{h \ln n} .
$$

Следовательно, $\mathbf{P}\left(\left|n-D_{n}\left(i_{3 / 4-\varepsilon}\right)-z_{n}^{(1)}\left(i_{3 / 4-\varepsilon}\right)\right| \geqslant n^{3 / 4-\psi}\right)>1 / 2-\mu$ для любых $\psi>0, \mu>0$ и при всех достаточно больших $n$. Наконец, в п. 3.4 было показано, что найдется такое число $c>0$, что

$$
\lim _{n \rightarrow \infty} \mathbf{P}\left(D_{n}\left(i_{3 / 4-\varepsilon}\right)-D_{n}\left(\sigma_{n}\right) \leqslant c n^{3 / 4-\varepsilon}\right)=1 .
$$

Поэтому для любых $\psi>0$ и $\mu>0$ при достаточно больших $n$

$$
\mathbf{P}\left(\left|n-D_{n}\left(\sigma_{n}\right)-z_{n}^{(1)}\left(i_{3 / 4-\varepsilon}\right)\right| \geqslant n^{3 / 4-\psi}\right)>\frac{1}{2}-\mu .
$$

Для завершения доказательства теоремы осталось показать, что последнее равенство влечет отличие от нуля всех частичных пределов последовательности $\left\{\mathbf{P}\left(\left|X_{n}-\mathbf{E} X_{n}\right| \geqslant n^{3 / 4-\psi}\right)\right\}_{n \in \mathbf{N}}$. Это верно, поскольку для любого $i \in\left\{t_{\alpha}(n), \ldots, i_{3 / 4-\varepsilon}\right\}$

$$
\begin{aligned}
\lim _{n \rightarrow \infty} \mathbf{P} & \left(n-D_{n}(i)-z_{n}^{(1)}(i)>R_{n}^{(1)}(i)\right) \\
\quad= & \lim _{n \rightarrow \infty} \mathbf{P}\left(n-D_{n}(i)-z_{n}^{(1)}(i)<-R_{n}^{(1)}(i)\right)=\frac{1}{2} .
\end{aligned}
$$

Автор признателен профессору А. В. Булинскому за постановку задачи, всестороннее обсуждение результатов и ценные замечания.

\section{СПИСОК ЛИТЕРАТУРЫ}

1. Alves O., Machado F., Popov S. Phase transition for the frog model. - Electron. J. Probab., 2002, v. 7, paper № 16, 21 p. 
2. Lebensztayn E., Machado F., Popov S. An improved upper bound for the critical probability of the frog model on homogeneous trees. - J. Statist. Phys., 2005, v. 119, № $1-2$, p. $331-345$.

3. A Alves O., Machado F., Popov S. The shape theorem for the frog model. - Ann. Appl. Probab., 2002, v. 12, № 2, p. 533-546.

4. Kurkova I., Popov S., Vachkovskaia M. On infection spreading and competition between independent random walks. - Electron. J. Probab., 2004, v. 9, paper № 11, p. 293-315.

5. Popov S. Frogs in random environment. - J. Statist. Phys., 2001, v. 102, № 1-2, p. 191-201.

6. Ramírez A.F., Sidoravicius V. Asymptotic behavior of a stochastic combustion growth process. - J. Eur. Math. Soc. (JEMS), 2004, v. 6, № 3, p. 293-334.

7. Telcs A., Wormald N. Branching and tree indexed random walks on fractals. - J. Appl. Probab., 1999, v. 36, № 4, p. 999-1011.

8. Durrett $R$. Random Graph Dynamics. Cambridge: Cambridge University Press, 2007, $212 \mathrm{p}$.

9. Bollobás B., Riordan O.M. Mathematical results on scale-free random graphs. Handbook of Graphs and Networks: From the Genome to the Internet. Ed. by S. Bornholdt and H. G. Schuster. Weinheim: Wiley-VCH, 2003, p. 1-34.

10. Machado F., Mashurian H., Matzinger H. CLT for the proportion of infected individuals for an epidemic model on a complete graph. - Markov Process. Related Fields, 2011, v. 17, № 2, p. 209-224.

11. Математическая энциклопедия, т. 5. М.: Советская энциклопедия, 1985.

12. Chernoff $H$. A measure of asymptotic efficiency for tests of a hypothesis based on the sum of observations. - Ann. Math. Statist., 1952, v. 23, № 4, p. 493-507.

13. Ширяев А. Н. Вероятность, т. 1. М.: МЦНМО, 2007, 551 с.

14. Петров В. В. Предельные теоремы для сумм независимых случайных величин. M.: Наука, 1987, 317 с.

15. Булинский А.В., Ширяев А.Н. Теория случайных процессов. М.: Физматлит, 2005, 402 c.

Поступила в редакцию

14.XI.2011

Исправленный вариант

13.VIII. 2012 